\title{
Caso 5/2010 - Homem de 41 Anos de Idade Portador de Hipertrofia Miocárdica com Evolução para Insuficiência Cardíaca Congestiva
}

\author{
Case 5/2010 - A 41 Year-old Man with Myocardial Hypertrophy who Evolved with Congestive Heart Failure \\ Rafael Moura de Almeida, Lucas José Tachotti Pires, José Soares Júnior, Wilson Ichiki, Wilson André Ichiki, Léa Maria \\ Macruz Ferreira Demarchi \\ Instituto do Coração (InCor) HC-FMUSP, São Paulo, SP - Brasil
}

Homem de 41 anos, natural e procedente da cidade de São Paulo, procurou atendimento em razão de dispneia rapidamente progressiva e edema generalizado.

O paciente sabia ser portador de hipertensão arterial e de hipertrofia de ventrículo esquerdo.

O paciente era assintomático até aos 33 anos de idade quando passou a apresentar dispneia aos esforços. Procurou atendimento médico na ocasião e foi diagnosticado aumento da área cardíaca. O quadro se manteve estável até aos 35 anos (maio 2001) quando apresentou episódio de palpitações taquicárdicas e dor precordial. Procurou atendimento médico e foi feito o diagnóstico de flutter atrial. O ecocardiograma (13 set 2001) revelou hipertrofia moderada de ventrículo esquerdo e derrame pericárdico moderado; a cineangiocoronariografia (18 out 2001) não revelou lesões obstrutivas nas artérias coronárias. Recebeu prescrição de amiodarona e foi encaminhado a este Hospital.

O exame físico (13 dez 2001) revelou frequência cardíaca 68 bpm, pressão arterial de 140/95 mmHg. O restante do exame físico não revelou outras alterações ou achados diagnósticos.

O eletrocardiograma (18 dez 2001) revelou ritmo sinusal, frequência 78 bpm, duração do PR 157 ms, do QRS 108 ms, do QT 414 ms, SÂQRS + 130 para trás. A análise morfológica revelou onda $\mathrm{P}$ de amplitude aumentada, QS em derivações I, aVL e onda Q em V6. Foram feitos os diagnósticos de sobrecarga atrial direita, área eletricamente inativa lateral e alterações da repolarização ventricular.

A avaliação laboratorial (19 dez 2001) revelou: hemoglobina $15,5 \mathrm{~g} / \mathrm{dl}$, hematócrito 46\%, colesterol total $242 \mathrm{mg} / \mathrm{dl}$ triglicérides $241 \mathrm{mg} / \mathrm{dl}$, glicemia de jejum $107 \mathrm{mg} / \mathrm{dl}$ e creatinina $1,3 \mathrm{mg} / \mathrm{dl}$.

\section{Palavras-chave}

Insuficiência cardíaca; hipertrofia ventricular esquerda; hipertensão; fibrilação atrial; amiloidose.

Editor da Seção: Alfredo José Mansur (ajmansur@incor.usp.br) Editores Associados: Desidério Favarato (dclfavarato@incor.usp.br) Vera Demarchi Aiello (anpvera@incor.usp.br)

Correspondência: Vera D. Aiello •

InCor - Av. Dr. Enéas de Carvalho Aguiar, 44 - 05403-000 - São Paulo, SP E-mail: anpvera@incor.usp.br
Foi suspenso o uso de amiodarona e prescrito atenolol.

O paciente evoluiu com piora da dispneia aos esforços. A piora foi associada ao uso de atenolol, o qual foi suspenso pelo paciente e voltou ao uso de amiodarona 200 mg/dia.

Foi realizado o teste ergométrico (21 fev 2003). Na condição basal a frequência cardíaca foi 124 bpm e a pressão arterial 134/98 mmHg; após 2 min e 45s de esforço elevaramse para 192 bpm e 158/94 mmHg. Apresentou fibrilação atrial e episódio de taquicardia ventricular não sustentada durante o esforço.

O eletrocardiograma de longa duração pelo sistema Holter (21 fev 2003) revelou ritmo de fibrilação atrial, 202 extrassístoles ventriculares isoladas e um episódio de taquicardia ventricular não sustentada com 4 batimentos e frequência de 155 bpm.

O ecocardiograma (2003) revelou aumento de átrios esquerdo e direito, hipertrofia septal assimétrica e fração de ejeção de ventrículo esquerdo normal (Tab. 1).

Foram prescritos enalapril $5 \mathrm{mg}$, amiodarona $200 \mathrm{mg}$ e varfarina $5 \mathrm{mg}$.

Os sintomas se atenuaram; permaneceu com dispneia desencadeada por grandes esforços e ocasionais episódios de palpitações durante 2 anos. Sucedeu então rápida intensificação da dispneia que passou a ocorrer mesmo com o paciente em repouso. Depois de um mês, não observando melhora, procurou atendimento médico.

O exame físico (3 jun 2004) revelou frequência cardíaca 98 bpm, 24 incursões respiratórias por minuto, pressão arterial 140/110 mmHg, aumento da pressão venosa jugular, estertores em base hemitórax esquerdo. O exame do coração revelou batimentos arrítmicos, bulhas hipofonéticas e sopro sistólico $+++/ 4+$ em área aórtica. O fígado foi palpado a $2 \mathrm{~cm}$ do rebordo costal direito e havia edema $++/ 4+$ em membros inferiores.

A radiografia de tórax revelou aumento da área cardíaca de configuração arredondada.

O eletrocardiograma (5 jun 2004) revelou flutter atrial, frequência cardíaca 79 bpm, baixa voltagem do complexo QRS no plano frontal, eixo QRS desviado para direita e anteriorizado e diminuição de potenciais de ventrículo esquerdo, sugestivo de sobrecarga ventricular direita e área eletricamente inativa parede lateral (Figura 1).

O ecocardiograma (3 jun 2004) revelou que as dimensões do ventrículo esquerdo e do átrio esquerdo eram semelhantes 


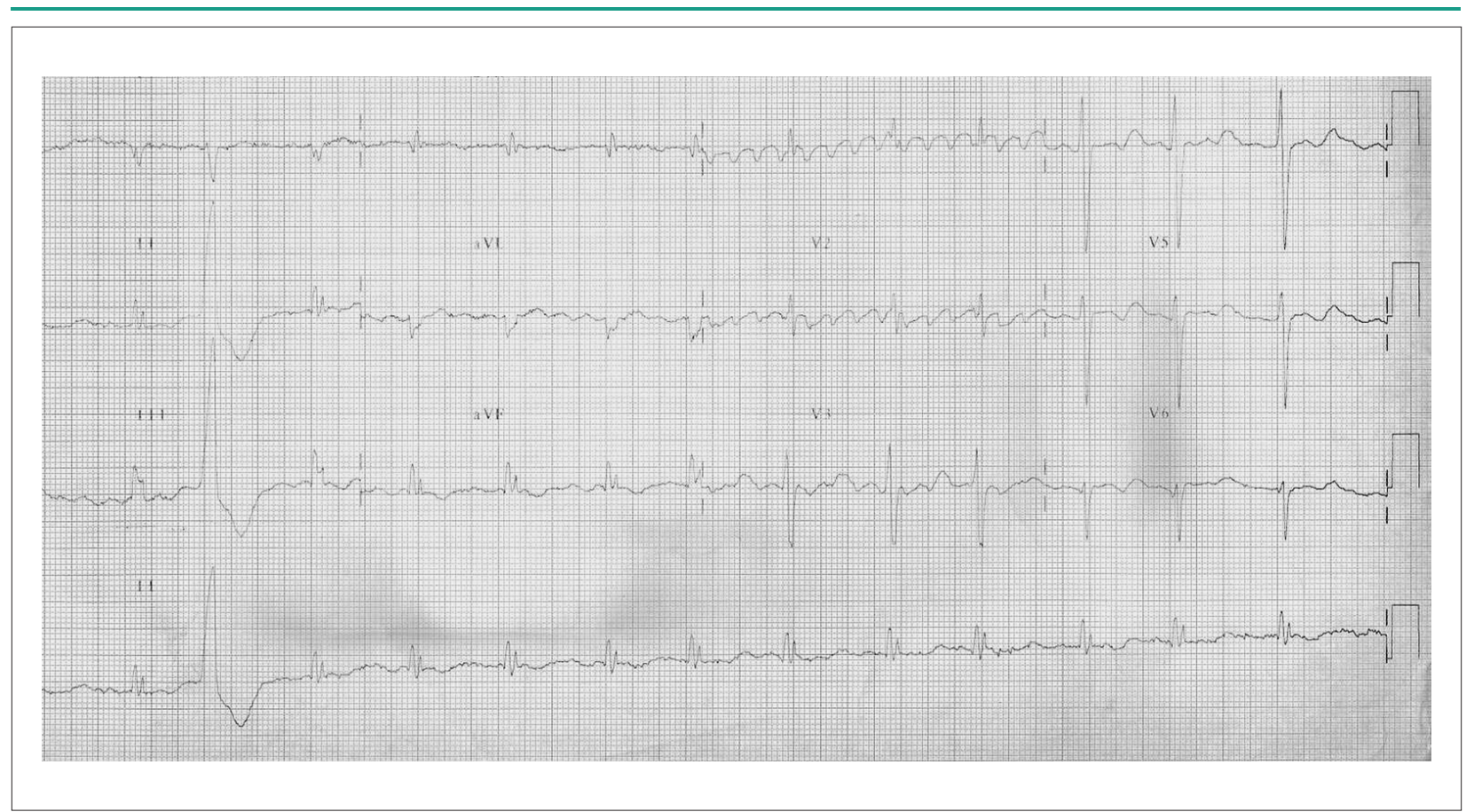

Fig. 1 - (ECG): flutter atrial e provável sobrecarga ventricular direita (SÂQRS para direita e anteriorizado).

às anteriormente medidas e hipertrofia do ventrículo direito e grande derrame pericárdico, sem sinais de restrição ou tamponamento cardíaco (Tab. 1).

Foram indicadas drenagem e biópsia pericárdica. Na intervenção (5 jun 2004) houve drenagem de $450 \mathrm{ml}$ de líquido citrino. O exame anatomopatológico dos fragmentos de pericárdio examinados revelou discreta fibrose e neovascularização do pericárdio, sem evidência de células neoplásicas ou infiltrado inflamatório.

O ecocardiograma de controle (15 jun 2004) revelou derrame pericárdico discreto.

Houve melhora da dispneia e o paciente recebeu alta hospitalar (15 jun 2004).

\section{Tabela 1 - Evolução ecocardiográfica}

\begin{tabular}{lcccc}
\hline & 2003 & $\mathbf{2 0 0 4}$ & $\mathbf{2 0 0 7}$ & 11 jun 08 \\
\hline Aorta (mm) & & 26 & & 32 \\
\hline Átrio esquerdo (mm) & 58 & 58 & 62 & 59 \\
\hline Septo (mm) & 23 & 19 & 20 & 22 \\
\hline Parede posterior (mm) & 12 & 13 & 12 & 12 \\
\hline Ventrículo esquerdo & & & & \\
\hline Diástole (mm) & 37 & 40 & 42 & 38 \\
\hline Sístole (mm) & 25 & 30 & 29 & 29 \\
\hline FEVE (\%) & 68 & & 56 & 48 \\
\hline Pressão sistólica VD (mmHg) & & 60 & & \\
\hline Ventrículo direito (mm) & & 40 & Dilatado & Dilatado \\
\hline
\end{tabular}

O mapeamento da espessura miocárdica por ecocardiograma (4 jun 2007) revelou as seguintes medidas dos segmentos: anterior (basal $17 \mathrm{~mm}$, médio $19 \mathrm{~mm}$ ), anterosseptal (basal $20 \mathrm{~mm}$, médio $22 \mathrm{~mm}$ ), inferosseptal (basal 15 mm, médio 16 mm), inferior (basal e médio 11 mm) e inferolateral (basal 12, médio $13 \mathrm{~mm}$ ), anterolateral (basal $11 \mathrm{~mm}$, médio $15 \mathrm{~mm}$ ), apical septal $14 \mathrm{~mm}$, apical lateral $14 \mathrm{~mm}$, calota apical $12 \mathrm{~mm}$.

O eletrocardiograma de alta resolução (26 jul 2007) revelou alargamento do QRS - duração de QRS padrão 140 ms; duração do QRS filtrado 130 ms; duração abaixo de $40 \mathrm{mV}$ 14 ms; e ausência de potenciais tardios de baixa amplitude nos $40 \mathrm{~ms}$ finais, $61,7 \mathrm{mV}$.

A dispneia aos esforços moderados permaneceu estável por mais 4 anos, até que em maio de 2008 progrediu até aparecer mesmo no repouso, no decúbito e surgir edema generalizado. Depois de 20 dias, como não houvesse melhora, procurou novamente atendimento médico (6 jun 2006).

O exame físico (6 jun 2008) revelou paciente em regular estado geral, com frequência de pulso de 90 bpm, pressão arterial 70/50 mmHg. A pressão venosa jugular estava elevada. A semiologia pulmonar foi normal. A semiologia cardíaca revelou hipofonese de bulhas e não havia sopros. O abdome era globoso e havia ascite, além de edema escrotal e de membros inferiores $++++/ 4+$.

O ECG (6 jun 08) revelou fibrilação atrial, frequência cardíaca 96 bpm, duração QRS 143 ms, baixa voltagem do complexo QRS, SÂQRS + 130 para frente, bloqueio do ramo direito, com R puro em V1 e V2, e área eletricamente inativa lateral (Figura 2). 


\section{Correlação Anatomo-clínica}

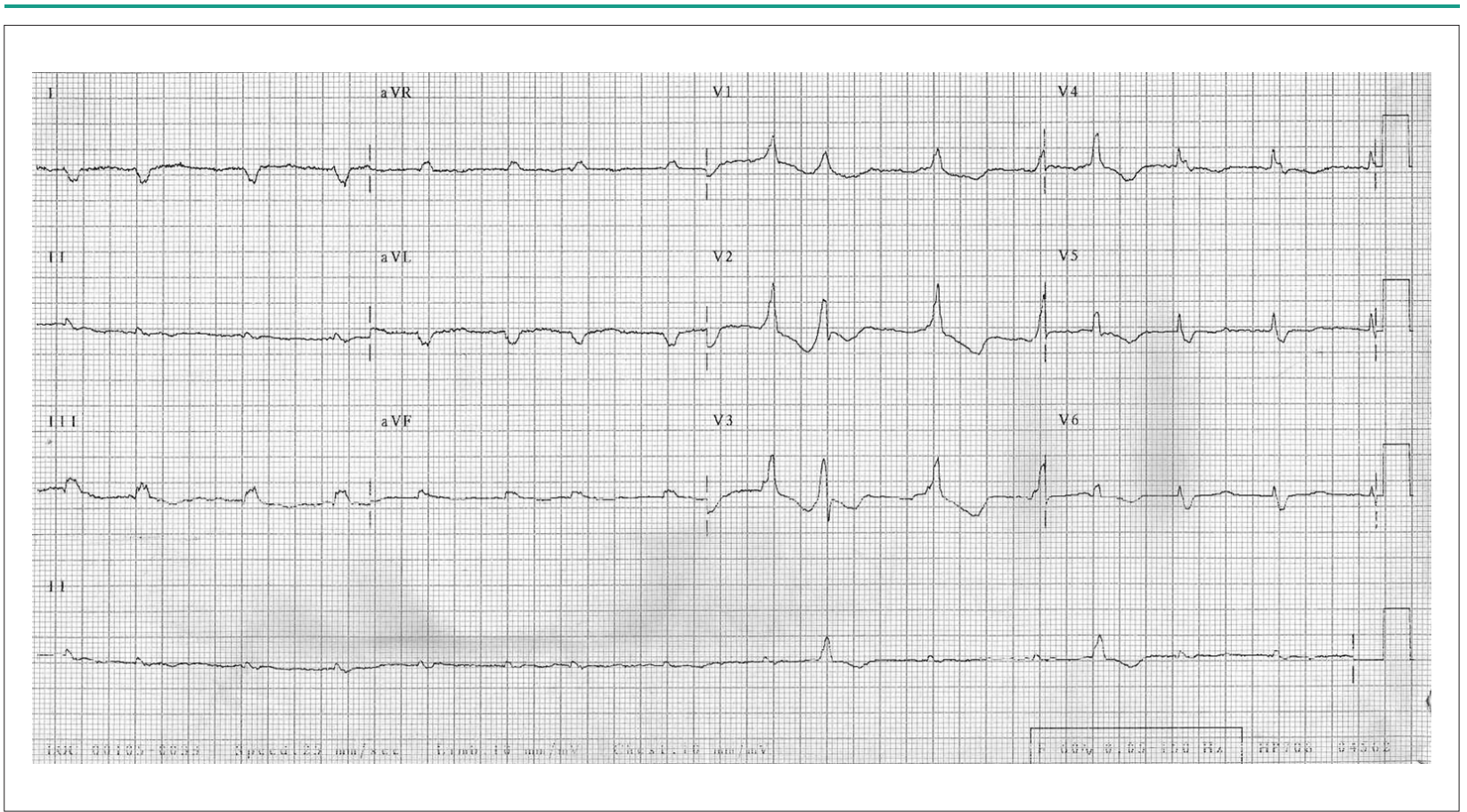

Fig. 2 - (ECG): fibrilação atrial, baixa voltagem plano frontal, QRS alargado, $R$ puros me $V_{1}$ e $V_{2}$.

A radiografia de tórax (6 jun 2008) revelou aumento global de área cardíaca sem sinais de congestão pulmonar.

Os exames laboratoriais (6 jun 08) revelaram hemoglobina $11,5 \mathrm{~g} / \mathrm{dl}$, hematócrito 34\%, leucócitos 6.700/ $\mathrm{mm}^{3}$ (68\% neutrófilos, $1 \%$ eosinófilos, $1 \%$ basófilos, 13\% linfócitos e $17 \%$ monócitos), plaquetas $161.000 / \mathrm{mm}^{3}$, ureia $67 \mathrm{mg} / \mathrm{dl}$, creatinina 2,3 mg/dl, potássio 3,6 mEq/l, sódio $136 \mathrm{mEq} / \mathrm{l}$, tempo de protrombina INR 2,7, relação de tempos de tromboplastina ativada 1,57 , creatinofosfoquinase fração $\mathrm{MB}$ massa 3,73 ng/ml e troponina I 0,21 ng/ml.

O ecocardiograma (11 jun 08) revelou hipocinesia do ventrículo esquerdo e direito, e de insuficiência tricúspide acentuada e derrame pericárdico moderado. (Tab. 1).

A ultrassonografia de abdome total (12 jun 08) revelou vias urinárias sem anormalidades, ectasia da veia cava inferior e hepáticas, ascite moderada, fígado com bordas rombas e textura finamente heterogênea.

Os exames laboratoriais (12 jun 08) revelaram creatinina 2,94 mg/dl, ureia $110 \mathrm{mg} / \mathrm{dl}$, potássio 5,9 mEq/l, sódio $134 \mathrm{mEq} / \mathrm{l}$.

A cintilografia de ventilação e de perfusão pulmonar (16 jun 08) foi considerada como de baixa probabilidade de tromboembolismo pulmonar.

A cintilografia cardíaca com pirofosfato de tecnécio (20 jun 2008) revelou captação discreta a moderada difusa no miocárdio (Figura 3 e 4).

A ressonância magnética cardíaca (27 jun 06) revelou dilatação biatrial acentuada; câmaras ventriculares de dimensões normais; disfunção ventricular esquerda discreta por hipocinesia difusa; função ventricular direita normal. Havia refluxo tricúspide acentuado e moderado derrame

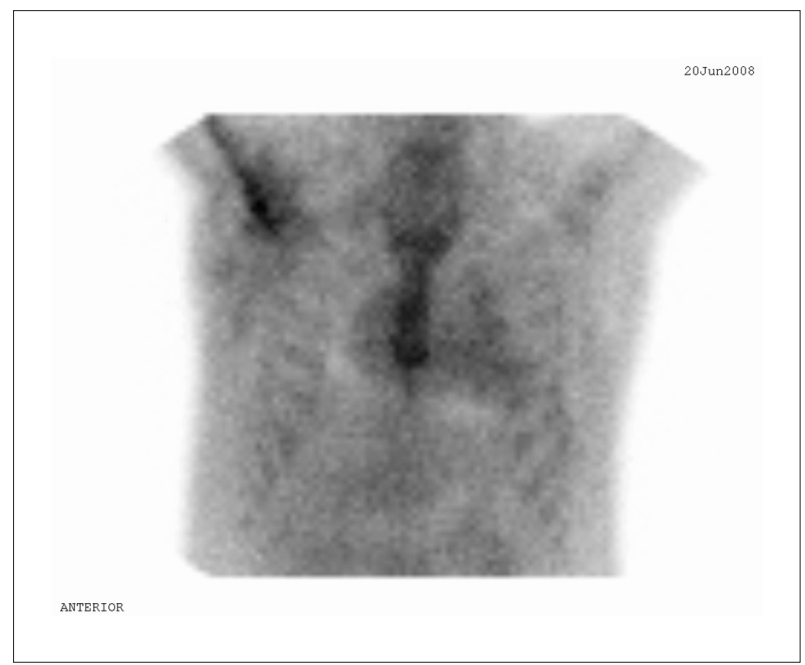

Fig. 3 - Cintilografia pirofostato de tecnécio em projeção anterior - captação do pirofostato em área cardíaca, além da captação mais intensa em esterno.

pericárdico, derrame pleural bilateral e não havia trombos. Os achados foram considerados compatíveis com amiloidose.

A pesquisa de proteína de Bence Jones na urina foi negativa.

Na evolução o ecocardiograma (11 jul 08) revelou derrame pericárdico acentuado (Tab. 1).

Apesar do tratamento com uso de diuréticos e inotrópicos por via intravenosa, houve piora acentuada do quadro com edema generalizado, derrame pleural e derrame pericárdico. Foi realizada outra drenagem pericárdica, além de paracentese bilateral do tórax sem intercorrências (18 jul 08). 


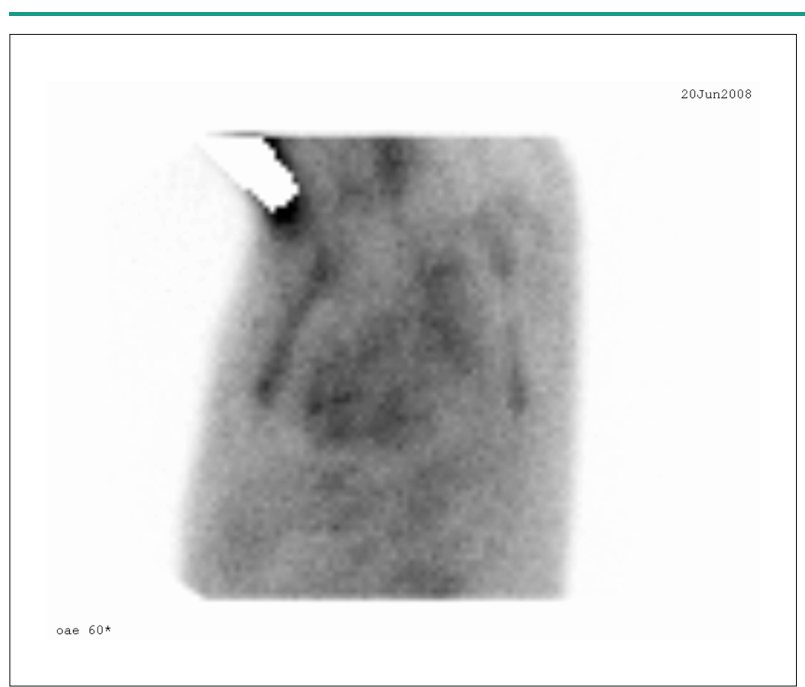

Fig. 4 - Cintilografia pirofostato de tecnécio em obliqua anterior esquerda $60^{\circ}$ - captação do pirofostato em área cardíaca, além da captação mais intensa em esterno.

O paciente apresentou tosse com expectoração purulenta. Foram identificados cocos gram positivos no líquido pleural esquerdo. Foi iniciada a antibioticoterapia com oxacilina e ceftriaxona (21 jul 08) substituídos no dia seguinte por tazobactam e vancomicina.

A ultrassonografia de abdome total (22/07/08) revelou hepatomegalia com forma e contornos normais e bordas rombas; as vias biliares intra e extra-hepáticas foram normais e havia esplenomegalia homogênea; ascite moderada. Os rins estavam normais.

Os exames laboratoriais (26 jul 2008) revelaram hemoglobina 10,5 g/dl, hematócrito 33\%, leucócitos 36400/ $\mathrm{mm}^{3}$ (3\% promielócitos, 2\% mielócitos, 8\% metamielócitos, $9 \%$ bastonetes, $63 \%$ segmentados, $2 \%$ eosinófilos, $1 \%$ basófilos, 3\% linfócitos e 9\% monócitos), plaquetas 102.000/ $\mathrm{mm}^{3}$, ureia $77 \mathrm{mg} / \mathrm{dl}$, creatinina $3 \mathrm{mg} / \mathrm{dl}$, bilirrubinas 7,8 $\mathrm{mg} / \mathrm{dl}$ (direta 6,6 mg/dl), fosfatase alcalina $107 \mathrm{UI} / \mathrm{l}$, gama GT $215 \mathrm{UI} / \mathrm{l}$, aspartato aminotransferase $47 \mathrm{UI} / \mathrm{ml}$, alanina aminotransferase $45 \mathrm{UI} / \mathrm{l}$, proteína C reativa $163 \mathrm{mg} / \mathrm{l}$, lactato arterial $52 \mathrm{mg} / \mathrm{dl}$, tempo de protrombina (INR) 2,1, relação TTPA 1,5, gasimetria arterial: $\mathrm{pH} 7,20, \mathrm{pCO}_{2} 39,5 \mathrm{mmHg}$, $\mathrm{pO}_{2} 75,7 \mathrm{mmHg}$, saturação de $\mathrm{O}_{2}, 92,5 \%$, bicarbonato 14,8 $\mathrm{mEq} / \mathrm{l}$ e excesso de base $-12,1 \mathrm{mEq} / \mathrm{l}$.

Apesar das medidas terapêuticas o paciente evoluiu em choque séptico a partir de 23 jul 2008, instalou-se falência de múltiplos órgãos e faleceu em assistolia (27 jul 2008).

\section{Aspectos clínicos}

O paciente, em seu quadro clínico inicial, aos 33 anos de idade, apresentou-se com queixa de dispneia desencadeada aos esforços. Nesse momento, havia importância em tentar definir a origem mais provável da queixa. Quando resultante de cardiopatia, a dispneia associa-se a aumento da pressão capilar pulmonar, com consequente congestão intersticial secundária. Outras potenciais causas seriam pulmonares, por alterações tireoideas, anemia, etc ${ }^{1,2}$. A presença de aumento de área cardíaca relatada na primeira radiografia de tórax aumenta, já nesse momento, a probabilidade de que a origem fosse cardíaca.

Trata-se, portanto, de paciente com insuficiência cardíaca (IC), que poderia ser decorrente de disfunção sistólica (fração de ejeção - FE - menor que 50\%) ou diastólica do ventrículo esquerdo (VE) (dificuldade anormal para o enchimento do VE na diástole $)^{3}$.

Disfunção diastólica é a causa de IC em 40 a 50\% dos pacientes. Para o diagnóstico de insuficiência cardíaca com fração de ejeção preservada (ICFEP) e disfunção diastólica é necessária a presença simultânea de três fatores: sinais ou sintomas de IC congestiva; FE de VE normal ou discretamente reduzida; e evidências objetivas de disfunção diastólica de VE: relaxamento e enchimento anormal do VE, distensibilidade diastólica anormal ou rigidez diastólica. As evidências de disfunção diastólica podem ser obtidas a partir de dados hemodinâmicos, níveis de peptídeos natriuréticos, dados ecocardiográficos e do Doppler tecidual ${ }^{4}$.

A evolução com dor precordial e palpitações, após 2 anos, sem alterações significativas no exame físico, com hipertrofia ventricular e fração de ejeção normal no ecocardiograma, além de derrame pericárdico e ausência de coronariopatia, remete-nos às possibilidades de doença cardíaca hipertensiva, miocardite acompanhada por acometimento pericárdico, cardiomiopatia hipertrófica $(\mathrm{CMH})$ e síndromes restritivas, como as doenças de depósito.

A doença cardíaca hipertensiva pode ser definida como resultado da sobrecarga imposta ao VE pelo aumento da pressão arterial e da resistência vascular periférica total. Nesse caso, trata-se de um paciente jovem com hipertrofia ventricular esquerda (HVE) evoluindo com ICFEP. Como consequência da HVE pode-se observar incidência aumentada de algumas arritmias, como extrassístoles ventriculares, arritmias ventriculares complexas ${ }^{5}$ e fibrilação atrial (FA), o que justificaria as taquiarritmias apresentadas pelo paciente.

Para o diagnóstico de HVE, o eletrocardiograma (ECG) é um método pouco sensível, porém bastante específico, entretanto os achados do paciente em discussão não preenchem esses critérios. Já o ecocardiograma é um método de baixo custo e é considerado o método não invasivo de eleição para o diagnóstico de aumento de massa cardíaca. Poder-se-ia pensar em investigação para hipertensão secundária por se tratar de um paciente jovem com HVE. Porém, nesse caso, uma vez que o paciente aparentemente obteve controle pressórico utilizando apenas $5 \mathrm{mg}$ de enalapril, portanto com fácil controle pressórico, pela evolução apresentada e pelo fato da hipertrofia ser claramente assimétrica, a hipótese de doença cardíaca hipertensiva poderia ser descartada ${ }^{6,7}$.

As miocardites, que são mais frequentemente causadas por agentes virais ${ }^{8}$, possuem um amplo espectro de apresentação clínica, que varia desde quadros subclínicos ou oligosintomáticos até a morte súbita cardíaca. A infecção por alguns agentes específicos pode estar associada a apresentações menos frequentes, como o fenótipo de cardiomiopatia hipertrófica sem disfunção ventricular inicialmente apresentado pelo caso em questão - que pode ser causado pelo vírus da hepatite $C$, sendo esse de maior 


\section{Correlação Anatomo-clínica}

prevalência em populações de países asiáticos ${ }^{9}$. Também na miocardite fulminante, caracterizada por apresentação aguda muito grave, inclusive com choque cardiogênico, observa-se, na avaliação ecocardiográfica, um aumento da espessura septal com menor aumento dos diâmetros ventriculares.

A presença de arritmias associadas às alterações miocárdicas é também frequente nas miocardites, necessitando muitas vezes de tratamento específico, e/ou sendo indicador de possível evolução desfavorável da doença, como ocorre na miocardite de células gigantes. Nesse tipo específico de inflamação miocárdica, entretanto, seria esperada uma história de menor duração - diferentemente do apresentado pelo caso -, uma vez que os pacientes apresentam malmau prognóstico, com sobrevida média de menos de 6 meses, e necessidade muito frequente de transplante cardíaco quando sobrevivem ao quadro agudo ${ }^{8}$. A miocardite crônica ativa, por sua vez, caracteristicamente teria uma evolução mais prolongada e insidiosa, em geral com manifestação fenotípica de cardiomiopatia dilatada, chegando a índices de mortalidade de 56\% em 4 anos. Pode ainda ocorrer acometimento pericárdico associado às miocardites ${ }^{8}$.

Outra hipótese para o caso são as cardiomiopatias restritivas e infiltrativas, que se caracterizam por limitação ao enchimento ventricular e rigidez ventricular, com ventrículos normais ou discretamente dilatados e dilatação atrial importante ${ }^{9}$. A espessura ventricular é normal ou aumentada, dependendo da etiologia. Os sintomas podem ser decorrentes da falência do ventrículo direito (VD), que se manifesta com aumento da pressão venosa jugular, edema periférico e ascite e/ou falência do VE, ocasionando dispneia e edema pulmonar ${ }^{10,11}$. A cardiomiopatia restritiva é a mais rara das cardiomiopatias, sendo a amiloidose sua forma mais comum ${ }^{12}$.

Como uma das causas de cardiomiopatia restritiva, a sarcoidose é uma doença granulomatosa sistêmica de etiologia desconhecida, caracterizada pelo envolvimento de vários tecidos por granulomas não $\operatorname{caseosos}^{13}$. O envolvimento cardíaco primário é infrequente e as manifestações clínicas aparecem em menos de 5\% dos pacientes e se caracterizam por distúrbios de condução, arritmias ventriculares, síncope e morte súbita. O envolvimento miocárdico direto pelos granulomas e tecido cicatricial pode manifestar-se em forma de cardiomiopatia dilatada ou restritiva, com curso progressivo ${ }^{14,15}$. O ECG é inespecífico, podendo apresentar anormalidades da onda T, bloqueios ou ondas Q patológicas. Outros achados incluem pericardite e cor pulmonale. O ecocardiograma pode revelar adelgaçamento das paredes ventriculares e aumento da ecogenicidade ${ }^{14,16}$. A ressonância magnética cardíaca (RMC) é um método altamente sensível e específico para o seu diagnóstico. O caso apresentado mostra apenas acometimento cardíaco sem as demais manifestações dessa doença, e a RMC não evidenciou alterações sugestivas, o que torna pouco provável que seja esse o diagnóstico do paciente ${ }^{17}$.

A doença de Fabry é um distúrbio hereditário com herança recessiva ligada ao cromossomo $X$, resultante da deficiência da enzima lisossômica alfa galactosidase A. Em sua forma clássica, caracteriza-se por envolvimento acentuado da pele, dos rins e do miocárdio, incluindo a manifestação de angina pectoris e infarto do miocárdio, embora na maioria das vezes as artérias coronárias tenham aspecto angiográfico normal. Há aumento da espessura do VE, com função sistólica preservada e discretas, disfunção diastólica e insuficiência mitral. A RMC é adequada para diferenciar a doença de Fabry de outras cardiomiopatias $^{10,11}$. O paciente em discussão não apresenta manifestações extracardíacas compatíveis com a doença e a RMC é sugestiva de outra doença.

A hemocromatose resulta do depósito de ferro em tecidos parenquimatosos, como coração, fígado, gônadas e pâncreas. Sua apresentação clássica é através do aparecimento de insuficiência cardíaca, cirrose hepática, disfunção erétil, diabetes mellitus e artrite. O envolvimento cardíaco leva ao padrão combinado de cardiomiopatia dilatada e cardiomiopatia restritiva com disfunções sistólica e diastólica. O ECG inclui alterações do segmento ST e da onda T e arritmias supraventriculares. O ecocardiograma revela espessamento de parede, dilatação e disfunção ventriculares. Os marcadores de cinética do ferro mostram-se alterados ${ }^{10,11}$. A RMC com aquisição com a técnica T2 estrela pode fechar o diagnóstico ${ }^{18}$. No paciente em questão, não há manifestações extracardíacas sugestivas e os exames complementares não são compatíveis com hemocromatose.

As doenças endomiocárdicas manifestam-se com fibrose endocárdica que oblitera o ápice dos ventrículos e as regiões subvalvares ${ }^{10,11}$. Como se pode observar, esse não é o padrão do paciente em discussão.

A amiloidose é uma doença de depósito que pode ser classificada de acordo com a natureza bioquímica da proteína amiloide em amiloidose primária, secundária, senil, familiar e amiloidose dos pacientes com insuficiência renal dialítica. Deve ser considerada como diagnóstico diferencial em qualquer paciente com mais de 40 anos com insuficiência cardíaca inexplicável, síndrome nefrótica, neuropatia periférica ou hepatopatia. A amiloidose secundária e a amiloidose dos pacientes renais crônicos raramente apresentam envolvimento cardíaco. No ECG são encontrados bloqueios atrioventriculares e complexos QRS de baixa voltagem, e o ECG de alta resolução tem valor na predição do risco de morte súbita, diferentemente do que ocorre nos casos de $\mathrm{CMH}$. De forma semelhante a essa outra doença, entretanto, a infiltração amiloide também predispõe a FA e arritmias ventriculares ${ }^{10,11,19,20}$.

O ecocardiograma mostra aumento da espessura miocárdica, que tem aspecto granuloso, dilatação de átrios, derrame pericárdico, pleural ou ambos e disfunção diastólica. O derrame pericárdico não evolui habitualmente para tamponamento cardíaco, e a presença de disfunção do VD é um preditor independente de mau prognóstico ${ }^{10,11}$. A cintilografia com pirofosfato de tecnécio mostra captação proporcional à intensidade do depósito amiloide ${ }^{11}$. Uma vez que esse radioisótopo é capaz de ligar-se ao cálcio, o exame pode estar alterado também em outras situações, como calcificações e outras doenças de depósito. A RMC tem alta sensibilidade e especificidade que pode variar de $75 \%$ a $94 \%$, quando é encontrado padrão caracterizado por realce global e heterogêneo subendocárdico tardio com gadolínio ${ }^{17,21,22}$, enquanto a biópsia caracteriza a substância amiloide e confirma a suspeita de amiloidose.

No caso em discussão, inicialmente o diagnóstico de amiloidose parece pouco provável pela idade do paciente e pelo ECG apresentado. Entretanto, na evolução do caso 
a mudança do padrão eletrocardiográfico aproxima-se mais daquele comum aos casos de amiloidose (baixa voltagem), não sendo possível, porém, estabelecer se isto não teria ocorrido apenas pelo derrame pericárdico. Ademais, a pesquisa da proteína de Bence Jones foi negativa. Por outro lado, o paciente apresenta síndrome restritiva grave e foi realizada cintilografia com pirofosfato e RMC que são compatíveis com esse diagnóstico. Dessa forma, seria possível pensar em amiloidose cardíaca, em sua forma primária, como o diagnóstico do caso apresentado.

Um diagnóstico diferencial da cardiomiopatia restritiva é a pericardite constritiva, caracterizada por fibrose pericárdica que impede o enchimento das câmaras cardíacas. Ocorre congestão venosa sistêmica e redução do índice cardíaco. O ECG revela, normalmente, alterações inespecíficas da onda T e redução da voltagem, além de sinais de sobrecarga atrial esquerda ou FA. A radiografia de tórax mostra aumento atrial direito e o ecocardiograma pode revelar calcificações pericárdicas e derrame pericárdico. Entretanto, no presente caso, foram observados aumento da espessura da parede ventricular, pressão sistólica de artéria pulmonar superior a $50 \mathrm{mmHg}$ e RMC sem espessamento pericárdico, sendo todas essas alterações sugestivas de síndrome restritiva, e não constritiva ${ }^{10,11}$.

Por fim, a CMH constitui a doença cardíaca genética de maior prevalência ${ }^{23}$ e a causa mais comum de morte súbita cardíaca em pessoas jovens ${ }^{24}$. É característico da doença um padrão assimétrico de hipertrofia, havendo mais frequentemente (em $70 \%$ a $75 \%$ dos casos) evidência ecocardiográfica de hipertrofia difusa do septo ventricular e da parede anterolateral, bem como descrição de hipertrofia de ventrículo direito em alguns pacientes ${ }^{25}$. As manifestações clínicas podem surgir em qualquer idade, sendo mais frequente o diagnóstico durante a adolescência e o início da vida adulta.

A desorganização da arquitetura miocárdica induzida pela doença, associada ao aumento da massa ventricular e a alterações presentes na microvasculatura coronariana levam a isquemia miocárdica silenciosa, com formação de áreas de fibrose, que podem corresponder a regiões eletricamente instáveis, de onde podem se originar arritmias ventriculares. Além disso, essas áreas de fibrose, associadas à hipertrofia regional (em geral, septal), podem levar a alterações no ECG, com sobrecarga atrial, por exemplo, ondas $\mathrm{R}$ em $\mathrm{V} 1$, diminuição de ondas $\mathrm{R}$ e surgimento de ondas $\mathrm{Q}$ em derivações de parede lateral (DI, aVL, V5 e V6), alterações de repolarização ventricular e, em caso de fibrose em parede lateral, desvio do eixo para a direita, como pode ser observado no paciente em questão. Na evolução da doença, pode ainda ocorrer, de modo compatível com esse caso, alargamento do $\mathrm{QRS}^{26}$.

Os pacientes podem apresentar obstrução à via de saída do VE, que está relacionada ao desenvolvimento do quadro clínico de insuficiência cardíaca, a maior taxa de óbito cardiovascular e a maior risco de morte súbita ${ }^{3}$, e que é reduzida com o uso de betabloqueadores, e aumentada, entre outras situações, na presença de extrassístoles ventriculares (EV) e na manobra de Valsalva.

O paciente em questão apresentou piora clínica após a suspensão de amiodarona e início do uso de atenolol. Em caso de ser $\mathrm{CMH}$ o diagnóstico, e portanto o uso de betabloqueador ter sido corretamente indicado, a suspensão da amiodarona pode ter se associado a um aumento na frequência de EV, com consequente aumento do gradiente subaórtico, podendo ter levado ao surgimento de componente obstrutivo na doença gerando, assim, a piora do sintoma de dispneia. Outra possível causa da piora com a suspensão da amiodarona teria sido a maior incidência de arritmias paroxísticas supraventriculares, como a FA, reconhecidamente associadas à piora clínica desses pacientes, com piora do prognóstico ${ }^{23,26}$. Sabe-se que a incidência de arritmias supraventriculares, incluindo FA, por registros em Holter, pode chegar a até $30 \%$ em adultos ${ }^{26}$. A presença de FA nessa população, mesmo no primeiro ou segundo episódio paroxístico, é capaz de gerar formação de trombos e fenômenos cardioembólicos, fazendo com que haja indicação de anticoagulação nesses pacientes, com baixo limiar para seu início ${ }^{24,27}$.

Dez a 15\% dos pacientes evoluem com insuficiência cardíaca limitante (classe funcional New York Heart Association III ou IV), e apenas 3\% manifestam doença avançada com FE de VE menor que $50 \%{ }^{28}$. Um estudo publicado em 2007 mostrou, entre 1.226 pacientes com diagnóstico de $\mathrm{CMH}$, prevalência de fenótipo do tipo restritivo de $1,5 \%{ }^{29}$. Os pacientes com o fenótipo estudado apresentaram mais disfunção diastólica e maior diâmetro atrial esquerdo, além de menor tolerância ao exercício e pior prognóstico, com sobrevida de 56\% em 5 anos - versus 90\% naqueles sem o fenótipo do tipo restritivo $(p=0,008)$ - tendo sido observados, em $42 \%$ dos casos, sinais de insuficiência cardíaca direita, como ascite, desconforto abdominal e edema. Houve, ainda, maior tendência ao desenvolvimento de FA ou flutter atrial e maiores taxas de acidente vascular cerebral. Pode-se observar que, durante sua evolução, o paciente ora relatado apresentou diversas características compatíveis com esse fenótipo da doença.

Diante da possibilidade de ocorrência de morte súbita, estudos prévios definiram marcadores de risco, utilizados na estratificação desses pacientes. Aqueles diagnosticados antes dos 14 anos de idade apresentam maior risco. Outros fatores incluem: história pessoal de síncope ou morte súbita revertida; história familiar de morte súbita; hipertrofia grave (maior que $30 \mathrm{~mm})^{30}$; presença de taquicardia ventricular não sustentada (TVNS) na monitorização por Holter; comportamento pressórico anormal ao esforço - queda da pressão arterial ou aumento menor que $25 \mathrm{mmHg}$ da pressão sistólica ${ }^{26}$. O ECG de alta resolução, a análise da dispersão do intervalo QT e da variabilidade da frequência cardíaca apresentam baixa acurácia preditiva ${ }^{31}$. No caso em questão, o paciente apresentou, entre os fatores de risco listados, TVNS na monitorização por Holter, e não teve uma curva pressórica que pudesse ser avaliada no teste ergométrico, posto o baixo trabalho realizado.

O quadro clínico teve um caráter progressivo, desde o seu diagnóstico, caracterizado por três episódios mais importantes de descompensação, com alterações clínicas, eletrocardiográficas e ecocardiográficas cada vez mais significativas. Em sua última internação, o paciente evoluiu com disfunção sistólica biventricular, derrame pericárdico acentuado, edema, ascite e choque cardiogênico, acompanhados por insuficiência renal, 


\section{Correlação Anatomo-clínica}

coagulopatia e infecção pulmonar complicada com choque séptico, culminando em óbito.

Considerando a idade da apresentação inicial, as alterações clínicas encontradas ao longo dos anos de evolução, as alterações nos exames subsidiários realizados e as características de cada uma das doenças apresentadas ao longo desta discussão, consideramos a $\mathrm{CMH}$ como a principal hipótese diagnóstica nesse caso.

\section{Dr. Rafael Moura de Almeida e} Dr. Lucas José Tachotti Pires

\section{Hipótese diagnóstica}

Síndrome restritiva secundária a cardiomiopatia hipertrófica.

Dr. Rafael Moura de Almeida e Dr. Lucas José Tachotti Pires

\section{Cintilografia}

Cintilografia com 99 mTc-pirofosfato de 20 de junho de 2008 (Figuras 3 e 4).

As imagens cintilográficas do tórax, realizadas 3 horas após a administração endovenosa do 99 mTc-pirofosfato, obtidas nas projeções anterior e oblíqua anterior esquerda de $60^{\circ}$ demonstram captação difusa na projeção cardíaca de grau discreto/moderado.

O 99mTc-pirofosfato liga-se ao cálcio intracelular, que se acumula nas células devido a alterações do sarcolema. É importante ressaltar que o miocárdio normal não apresenta captação de $99 \mathrm{mTc}$-pirofosfato. O exame era muito utilizado no passado para avaliação de infarto agudo do miocárdio, onde o padrão clássico é de captação focal na parede acometida. A captação difusa é um achado infrequente e pouco específico, geralmente relacionado a doenças que acometem o coração difusamente como amiloidose, sarcoidose, perimiocardites, endocardites ou infarto subendocárdico. Na amiloidose o grau de captação está relacionado com o grau de espessamento do miocárdio, portanto com implicações prognósticas ${ }^{32,33}$. No caso em questão, a captação difusa em cardiomiopatia hipertrófica não é esperada, podendo estar relacionada aos achados da necropsia de infarto subendocárdico e/ou pericardite.

\section{Dr. José Soares Junior, Dr. Wilson Ichiki} e Dr. Wilson André Ichiki

\section{Necropsia}

À abertura do saco pericárdico houve saída de cerca de $250 \mathrm{ml}$ de sangue e coágulos, observando-se pericardite fibrinosa, difusa. O coração pesou 510 g (normal: 300-350 g) e exibia aumento do volume das quatro câmaras cardíacas. Havia dilatação moderada do ventrículo direito e dos átrios. Os ventrículos apresentavam hipertrofia moderada e simétrica, com relação entre as espessuras do septo ventricular e da parede livre do ventrículo esquerdo $=1,2$, sem obstrução cavitária, notando-se extensas áreas de fibrose no miocárdio do ventrículo esquerdo (Figuras 5 e 6). O endocárdio de ambos os ventrículos era delgado, liso e brilhante. Na aurícula direita havia um pequeno trombo misto, em organização. Não foram encontradas anormalidades nas valvas atrioventriculares ou ventrículo-arteriais. O estudo das artérias coronárias evidenciou trajeto intramiocárdico ("ponte miocárdica") do $2^{\mathrm{O}}$ ao $4 \underline{\mathrm{O}} \mathrm{cm}$ do ramo interventricular esquerdo (Figura 7) e, à histologia, havia aterosclerose coronária leve, com obstrução máxima de $30 \%$ da luz das artérias coronárias principais.

Cortes histológicos dos ventrículos mostraram hipertrofia miocárdica difusa, desarranjo espacial de cardiomiócitos

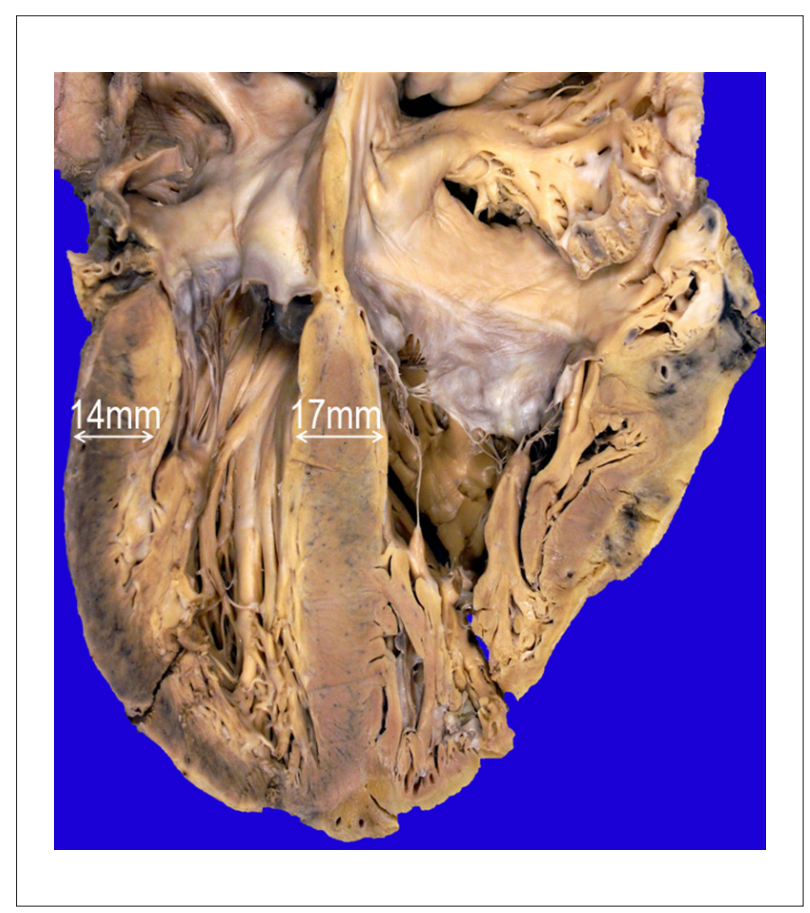

Fig. 5 - Corte do coração do tipo quatro câmaras: hipertrofia simétrica dos ventrículos esquerdo e direito, sem obstrução cavitária. Relação entre espessura do septo ventricular/espessura da parede lateral livre do ventrículo esquerdo $=17 \mathrm{~mm} / 14 \mathrm{~mm}=1,2$.

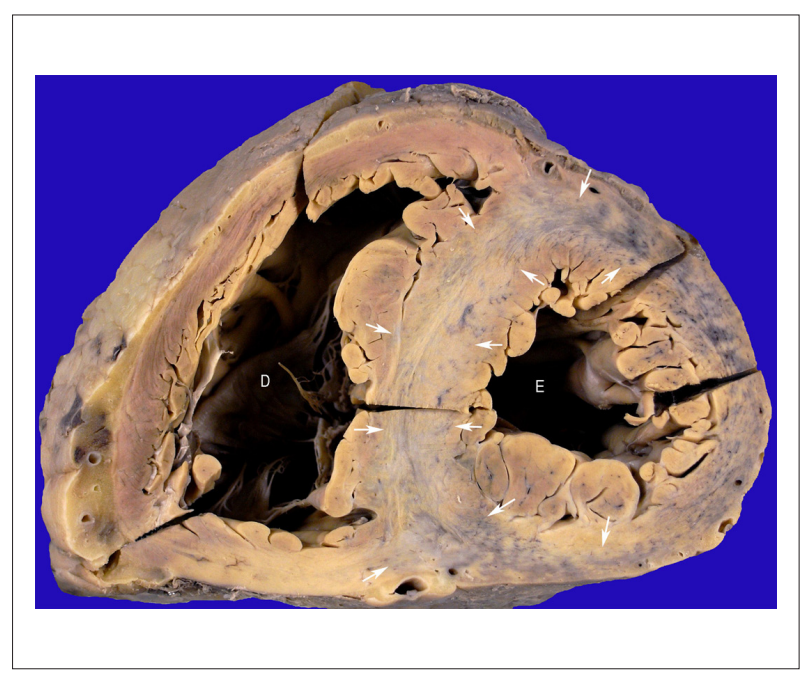

Fig. 6 - Corte transversal dos ventrículos: hipertrofia miocárdica dos ventrículos esquerdo e direito e fibrose acentuada no miocárdio do ventrículo esquerdo (entre setas). 


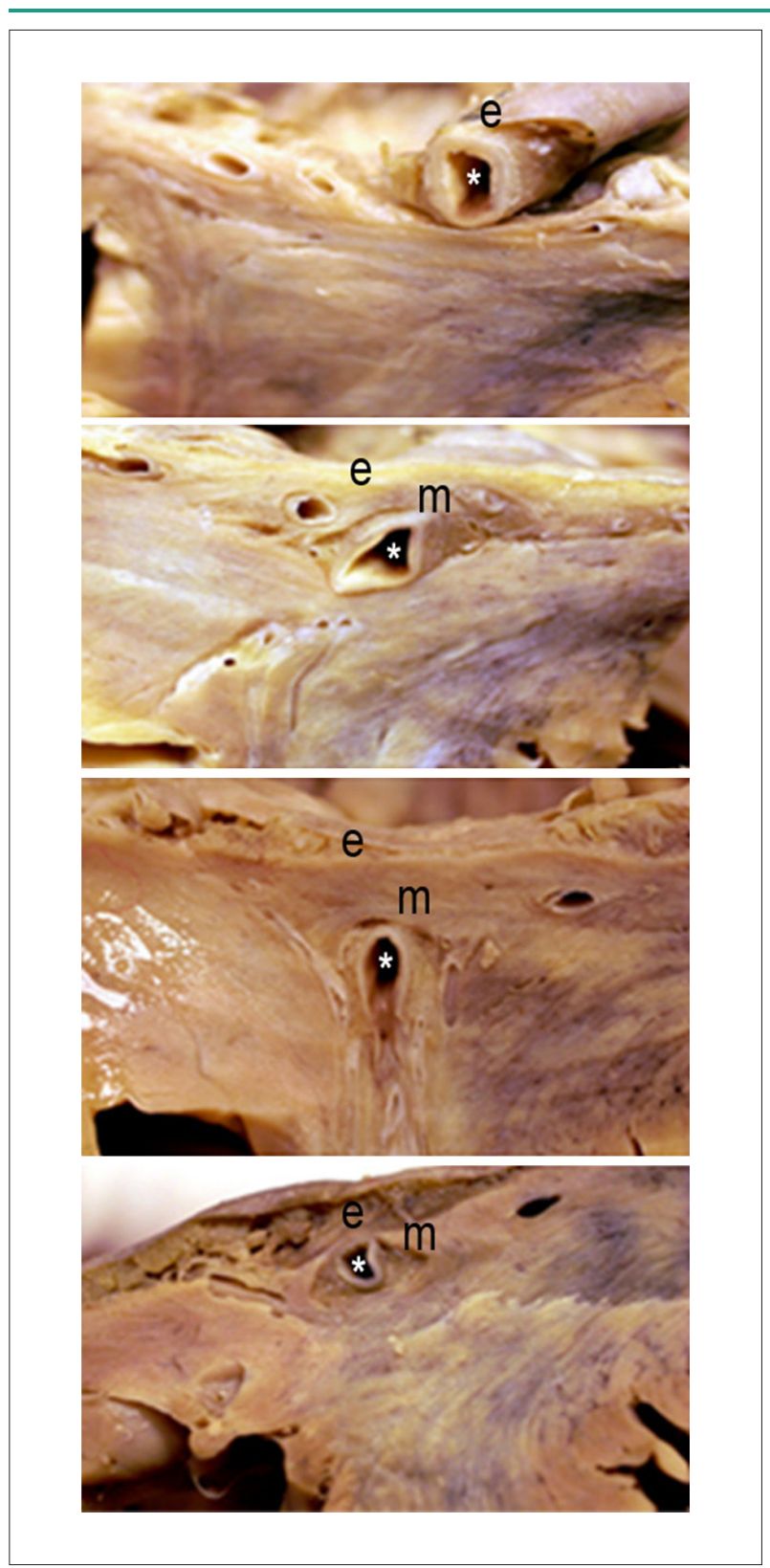

Fig. 7 - Cortes transversais dos ventrículos: trajeto intramiocárdico do ramo interventricular anterior da artéria coronária esquerda (e - endocárdio; $m$ miocárdio; * - luz coronária).

e de feixes de fibras cardíacas em mais de $20 \%$ das áreas amostradas, fibrose intersticial difusa desde a região subendocárdica até a subepicárdica, e espessamento da parede das artérias coronárias intramurais devido à proliferação mediointimal de células musculares lisas (figura 8). Embora presentes em ambos os ventrículos, essas alterações eram mais acentuadas no septo ventricular e na parede livre do ventrículo esquerdo. As pesquisas histoquímicas de substância amilóide e de glicogênio no miocárdio resultaram negativas. Diante dos achados anatomopatológicos da necropsia, fez-se o diagnóstico de cardiomiopatia hipertrófica, forma restritiva, sem obstrução da via de saída. Observou-se, ainda, congestão passiva crônica em pulmões, fígado e baço e edema generalizado de tecido subcutâneo, consequentes à insuficiência cardíaca congestiva.

Nos demais órgãos foram encontradas alterações secundárias ao choque, representadas por dano alveolar difuso e hemorragia intralveolar nos pulmões, necrose tubular aguda nos rins, necrose hepática centrilobular e infartos subendocárdicos recentes, pequenos e multifocais. Nos pulmões havia áreas localizadas de broncopneumonia em fase inicial. Porém, não foram observadas alterações morfológicas sugestivas de infecção em outros órgãos. A presença de sangue e coágulos no saco pericárdico pareceu ser mais um evento final, resultado das manobras de ressuscitação.

A causa do óbito foi falência de múltiplos órgãos decorrente de choque, predominantemente cardiogênico e agravado pela infecção pulmonar.

Léa Maria Macruz Ferreira Demarchi

\section{Diagnósticos anatomopatológicos}

1) Cardiomiopatia hipertrófica simétrica, não obstrutiva; 2) Insuficiência cardíaca congestiva; 3) Choque misto (cardiogênico/infeccioso); 4) Falência de múltiplos órgãos.

Léa Maria Macruz Ferreira Demarchi

\section{Comentário}

A cardiomiopatia hipertrófica $(\mathrm{CMH})$ é definida como cardiomiopatia primária genética, caracterizada morfologicamente por hipertrofia não dilatada do ventrículo esquerdo, na ausência de outra doença sistêmica ou cardíaca capaz de causar tal alteração ventricular, como por exemplo, a hipertensão arterial sistêmica e a estenose de valva aórtica ${ }^{34}$.

Tem espectro clínico abrangente, exibindo grande variabilidade morfológica, de sintomas e risco de complicações como morte súbita e insuficiência cardíaca ${ }^{35}$.

As principais características anatômicas da $\mathrm{CMH}$ foram obtidas a partir de dados de observação de necropsias e são assim definidas ${ }^{34}$ : hipertrofia miocárdica, desarranjo espacial de cardiomiócitos, artérias coronárias intramurais com alterações obstrutivas e fibrose miocárdica.

A hipertrofia miocárdica pode ser assimétrica ou simétrica. A forma de apresentação mais comum é a assimétrica, caracterizada por hipertrofia mais acentuada do septo ventricular, sendo que a relação entre as espessuras do septo ventricular/parede lateral do ventrículo esquerdo deve ser igual ou maior que 1,336. Quando localizada na porção basal do septo ventricular, a hipertrofia causa obstrução da via de saída do ventrículo esquerdo e, consequentemente, há espessamento da cúspide anterior mitral e fibrose endocárdica septal subaórtica. Devido ao atrito constante entre o folheto valvar e o endocárdio septal, a fibrose endocárdica pode adquirir o formato de imagem em espelho da cúspide anterior mitral. A forma simétrica caracteriza-se por hipertrofia concêntrica do ventrículo esquerdo, sem obstrução da via de saída ventricular.

$\mathrm{Na} \mathrm{CMH}$, o desarranjo espacial de cardiomiócitos deve estar presente em mais de $20 \%$ das áreas histologicamente amostradas $^{37}$ e caracteriza-se por conexões intercelulares 


\section{Correlação Anatomo-clínica}

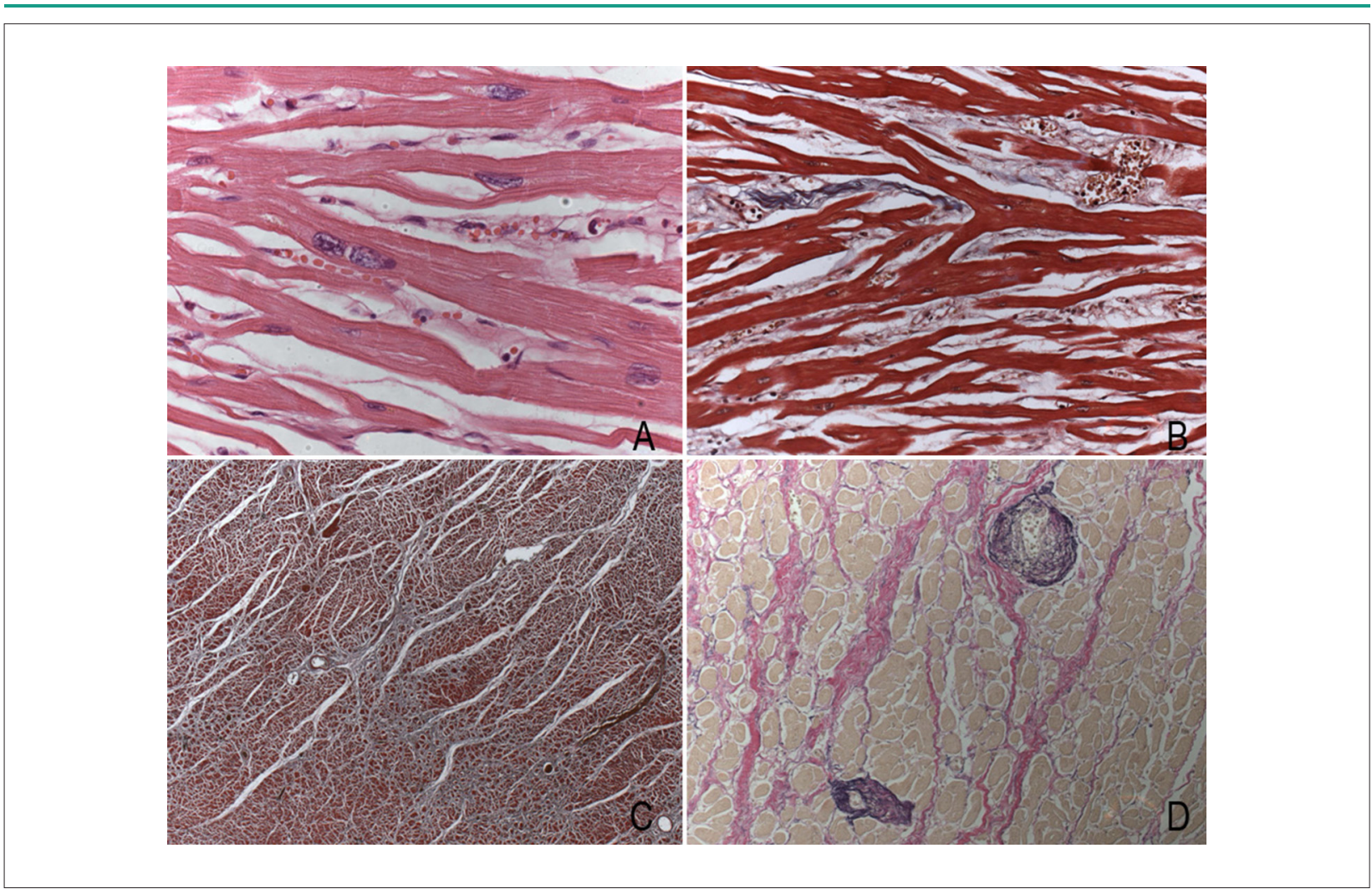

Fig. 8 - Microscopia óptica de cortes histológicos do miocárdio do ventrículo esquerdo: A- Hipertrofia miocárdica (hematoxilina-eosina, lente objetiva 40x). B- Desarranjo espacial de cardiomiócitos (tricrômico de Masson, lente objetiva 20x). C- Fibrose miocárdica difusa (áreas em azul pelo tricrômico de Masson, lente objetiva 2,5x). DDiminuição da luz e espessamento das túnicas íntima e média em parede de ramos intra-miocárdicos de artérias coronárias (coloração de Miller para fibras elásticas, lente objetiva 20x).

múltiplas e caóticas, sob a forma de ângulos retos e oblíquos ${ }^{34}$. Pode ser encontrado em qualquer parede de ambos os ventrículos e, mais frequentemente, ocupa grandes porções do ventrículo esquerdo. Áreas extensas de desarranjo são descritas em pacientes jovens que morreram em consequência da doença ${ }^{34}$.

As artérias coronárias intramurais apresentam alterações obstrutivas caracterizadas por espessamento de parede e estreitamento luminal, devido ao aumento de deposição de colágeno nas túnicas íntima e média. Tais alterações, associadas à desproporção entre a hipertrofia da massa ventricular e a circulação coronária, resultam em isquemia miocárdica e consequente fibrose, que pode ser focal ou transmural ${ }^{34}$.

Outro achado frequente na $\mathrm{CMH}$ é o trajeto intramural ou "ponte miocárdica" do ramo interventricular anterior da artéria coronária esquerda, que também foi observado nesse doente, mas seu significado fisiopatológico ainda não está totalmente esclarecido ${ }^{38}$

A contribuição da biópsia endomiocárdica (BEM) para o diagnóstico da cardiomiopatia hipertrófica é bastante limitada e, no caso em discussão, não mostrou desarranjo miocárdico, apenas hipertrofia de cardiomiócitos. $\mathrm{Na} \mathrm{CMH}$, as áreas de desarranjo localizam-se frequentemente em regiões mais profundas da parede ventricular, distantes da região subendocárdica, e, portanto, fora do alcance do biótomo. Porém, a BEM se mostra útil para o diagnóstico diferencial entre outras causas de hipertrofia ventricular, como amiloidose, doença de Pompe e, até mesmo, neoplasias ${ }^{37,39}$.

Os achados anatomopatológicos obtidos pela necropsia, associados aos dados clínicos e de exames complementares, foram fundamentais para o diagnóstico de $\mathrm{CMH}$ nesse doente.

\section{Dra. Léa Maria Macruz Ferreira Demarchi}

\section{Referências}

1. Romano MM, Maciel BC. História clínica. In: Serrano Jr CV, Timerman A, Stefanini E, editores. Tratado de cardiologia SOCESP. 2aㅡ ed. Barueri (SP): Manole; 2009. p. 211-22.

2. Ingram Jr RH, Braunwald E. Dyspnea and pulmonary edema. In: Braunwald

E, Fauci AS, Kasper DL, Hauser SL, Longo DL, Jameson JL, editors. Harrison`s principles of internal medicine. New York: McGraw-Hill; 2001. p. 199-203.

3. Maron MS, Olivotto I, Betocchi S, Casey SA, Lesser JR, Losi MA, et al. Effect of left ventricular outflow tract obstruction on clinical outcome in hypertrophic 
cardiomyopathy. N Engl J Med. 2003; 348 (4): 295-303.

4. Bocchi EA, Marcondes-Braga FG, Ayub-Ferreira SM, Rohde LE, Oliveira WA Almeida DR, et al / Sociedade Brasileira de Cardiologia. III Diretriz brasileira de insuficiência cardíaca crônica. Arq Bras Cardiol. 2009; 92 (6 supl.1): 1-71.

5. Messerli FH. Left ventricular hypertrophy, arterial hypertension and sudden death. J Hypertens. 1990; 8 (Suppl. 7): 181-6.

6. Póvoa RMS, Bombig MTN. Cardiopatia hipertensiva. In: Serrano Jr CV, Timerman A, Stefanini E, editores. Tratado de cardiologia SOCESP. $2^{\mathrm{a}}$ ed. Barueri (SP): Manole; 2009. p. 777-88.

7. Victor RG, Kaplan NM. Sysmic hypertension: mechanisms and diagnosis. In: Libby P, Bonow RO, Mann LD, Zipes PD, editors. Braunwald's heart disease: a textbook of cardiovascular medicine. 8th ed. Philadelphia: Elsevier Saunders; 2008. p. 1027-48.

8. Cardoso JN, Pereira-Barreto AC. Miocardites. In: Serrano Jr CV, Timerman A, Stefanini E, editores. Tratado de cardiologia SOCESP. $2^{2}$ ed. Barueri (SP): Manole; 2009. p. 1146-52.

9. Liu PP, Schultheiss HP. Myocarditis. In: Libby P, Bonow RO, Mann DL, Zipes DP, editors. Braunwald's heart disease. 8th ed. Philadelphia: Elsevier Saunders; 2008. p. 1775-92.

10. Gimenes VML. Cardiomiopatias restritivas e infiltrativas. In: Serrano Jr CV Timerman A, Stefanini E, editores. Tratado de cardiologia SOCESP. 2a ed. Barueri (SP): Manole; 2009. p. 1208-21.

11. Hare JM. The dilated, restrictive and infiltrative cardiomyopathies. In: Libby P, Bonow RO, Mann LD, Zipes PD, editors. Braunwald's heart disease: a textbook of cardiovascular medicine. 8th ed. Philadelphia: Elsevier Saunders; 2008. p. 1739-62.

12. Kushwaha SS, Fallon JT, Fuster V. Restrictive cardiomyopathy. N Engl J Med. 1997; 336 (4): 267-77.

13. Sharma OP. Diagnosis of cardiac sarcoidosis: an imperfect science, a hesitant art. Chest. 2003; 123 (1): 18-9.

14. Yazaki Y, Isobe $M$, Hiramitsu S, Hiroe M, Omichi C, Nakano T, et al. Comparison of clinical features and prognosis of cardiac sarcoidosis and idiopathic dilated cardiomyopathy. Am J Cardiol. 1998; 82 (4): 537-40.

15. Pisani B, Taylor DO, Mason JW. Inflammatory myocardial diseases and cardiomyopathies. Am J Med. 1997; 102 (5): 459-69.

16. Shimada T, Shimada K, Sakane T, Ochiai K, Tsukihashi H, Fukui M, et al. Diagnosis of cardiac sarcoidosis and evaluation of the effects of steroid therapy by gadolinium-DTPA-enhanced magnetic resonance imaging. Am J Med. 2001; 110 (7): 520-7.

17. Smedema JP, Snoep G, van Kroonenburgh MP, van Geuns RJ, Dassen WR, Gorgels AP, et al. Evaluation of the accuracy of gadolinium-enhanced cardiovascular magnetic resonance in the diagnosis of cardiac sarcoidosis. J Am Coll Cardiol. 2005; 45 (10): 1683-90.

18. Schmidt A, Rochitte CE. Ressonância magnética cardíaca. In: Serrano Jr CV, Timerman A, Stefanini E, editores. Tratado de cardiologia SOCESP. 2 $\underline{-}$ ed. Barueri (SP): Manole; 2009. p. 437-52

19. Selvanayagam JB, Hawkins PN, Paul B, Myerson SG, Neubauer S. Evaluation and management of the cardiac amyloidosis. J Am Coll Cardiol. 2007; 50 (22): $2101-10$

20. Falk RH. Diagnosis and management of the cardiac amyloidoses, Circulation. 2005; 112: 2047-60

21. Kwong RY, Falk RH. Cardiovascular magnetic resonance in cardiac amyloidosis. Circulation. 2005; 111 (2): 122-4.

22. Vogelsberg H, Mahrholdt H, Deluigi CC, Yilmaz A, Kispert EM, Greulich $\mathrm{S}$, et al. Cardiovascular magnetic resonance in clinically suspected cardiac amyloidosis: noninvasive imaging compared to endomyocardial biopsy. J Am
Coll Cardiol. 2008; 51 (10): 1022-30.

23. Bregagnollo EA, Carvalho FC. Cardiomiopatia hipertrófica. In: Serrano Jr CV Timerman A, Stefanini E, editores. Tratado de cardiologia SOCESP. 2 ${ }^{\mathrm{a}}$ ed. Barueri (SP): Manole; 2009. p. 1181-207.

24. Maron BJ. Hypertrophic cardiomyopathy. In: Libby P, Bonow RO, Mann DL, Zipes DP, editors. Braunwald's heart disease. 8th ed. Philadelphia: Elsevier Saunders; 2008. p. 1763-74.

25. Connolly HM, Oh JK. Echocardiography. In: Libby P, Bonow RO, Mann DL, Zipes DP, editors. Braunwald's heart disease. 8th ed. Philadelphia: Elsevier Saunders; 2008. p. 227-325.

26. McKenna WJ, Behr ER. Hypertrophic cardiomyopathy: management, risk stratification, and prevention of sudden death. Heart. 2002; 87 (2): 169-76.

27. Maron BJ, McKenna WJ, Danielson GK, Kappenberger LJ, Kuhn HJ, Seidman CE, et al. American College of Cardiology/European Society of Cardiology Clinical Expert Consensus Document on Hypertrophic Cardiomyopathy. A report of the American College of Cardiology Foundation Task Force on Clinical Expert Consensus Documents and the European Society of Cardiology Committee for Practice Guidelines. Eur Heart J. 2003; 24 (21): 1965-91.

28. Harris KM, Spirito P, Maron MS, Zenovich AG, Formisano F, Lesser JR, et al. Prevalence, clinical profile, and significance of left ventricular remodeling in the end-stage phase of hypertrophic cardiomyopathy. Circulation. 2006; 114 (3): 216-25.

29. Kubo T, Gimeno JR, Bahl A, Steffensen U, Steffensen M, Osman E, et al. Prevalence, clinical significance, and genetic basis of hypertrophic cardiomyopathy with restrictive phenotype. J Am Coll Cardiol. 2007;49 (25): 2419-26.

30. Spirito P, Bellone P, Harris KM, Bernabo P, Bruzzi P, Maron BJ. Magnitude of left ventricular hypertrophy and risk of sudden death in hypertrophic cardiomyopathy. N Engl J Med. 2000; 342 (24): 1778-85.

31. Piva e Mattos B. Estratificação de risco para morte súbita na cardiomiopatia hipertrófica: bases genéticas e clínicas. Arq Bras Cardiol. 2006; 87 (3): 391-9.

32. Taki J, Taki S, Ichiyanagi K, Akashi Y, Hisada K. Acute subendocardial infarction with diffuse intense TC-99m PYP uptake and minimal TI-201 abnormality. Clin Nucl Med. 1992; 17 (8): 643-5.

33. Yazaki Y, Isobe M, Hayasaka M, Tanaka M, Fujii T, Sekiguchi M. Cardiac sarcoidosis mimicking hypertrophic cardiomyopathy: clinical utility of radionuclide imaging for differential diagnosis. Jpn Circ J. 1998; 62 (6): 465-8.

34. Maron BJ, Towbin JA, Thiene G, Antzelevitch C, Corrado D, Arnett D, et al. Contemporary definitions and classification of the cardiomyopathies: an American Heart Association Scientific Statement from the Council on Clinical Cardiology, Heart Failure and Transplantation Committee; Quality of Care and Outcomes Research and Functional Genomics and Translational Biology Interdisciplinary Working Groups; and Council on Epidemiology and Prevention. Circulation. 2006; 113 (14): 1807-16.

35. Varnava AM, Elliott PM, Mahon N, Davies MJ, McKenna WJ. Relation between myocyte disarray and outcome in hypertrophic cardiomyopathy. Am J Cardiol. 2001; 88 (3): 275-9.

36. Maron BJ, Anan TJ, Roberts WC. Quantitative analysis of the distribution of cardiac muscle cell disorganization in the left ventricular wall of patients with hypertrophic cardiomyopathy. Circulation. 1981; 63 (4): 882-94.

37. Davies MJ, McKenna WJ. Hypertrophic cardiomyopathy: an introduction to pathology and pathogenesis. Br Heart J. 1994; 72 (6 Suppl.): S2-3.

38. Basso C, Thiene G, Mackey-Bojack S, Frigo AC, Corrado D, Maron BJ. Myocardial bridging, a frequent component of the hypertrophic cardiomyopathy phenotype, lacks systematic association with sudden cardiac death. Eur Heart J. 2009; 30 (13): 1627-34.

39. Edwards WD. Cardiomyopathies. Hum Pathol. 1987; 18 (6): 625-35. 